\title{
UNIFORMLY DISTRIBUTED MEASURES HAVE BIG PIECES OF LIPSCHITZ GRAPHS LOCALLY
}

\author{
A. Dali Nimer \\ University of Chicago, Department of Mathematics \\ 5734, S. University Ave., Chicago, IL 60637, U.S.A.; nimer@uchicago.edu
}

\begin{abstract}
The study of uniformly distributed measures was crucial in Preiss' proof of his theorem on rectifiability of measures with positive density. It is known that the support of a uniformly distributed measure is an analytic variety. In this paper, we provide quantitative information on the rectifiability of this variety. Tolsa had already shown that $n$-uniform measures have big pieces of Lipschitz graph(BPLG). Here, we prove that a uniformly distributed measure has BPLG locally.
\end{abstract}

\section{Introduction}

Understanding the geometry of uniformly distributed measures has been an important question in geometric measure theory ever since Preiss proved his remarkable theorem on the $n$-rectifiability of measures in $[\mathrm{P}]$. This theorem states that, given a Radon measure $\sigma$ in $\mathbf{R}^{d}$, if the $n$-density of $\sigma$

$$
\Theta^{n}(x, \sigma)=\lim _{r \rightarrow 0} \frac{\sigma(B(x, r))}{r^{n}}
$$

exists, is finite and positive $\sigma$-almost everywhere on $\mathbf{R}^{d}$, then there exists a countably $n$-rectifiable set $E$ such that $\sigma\left(\mathbf{R}^{d} \backslash E\right)=0$. The proof of Preiss' theorem relied heavily on the study of uniformly distributed measures. Indeed, these measures appear as blow ups (zoom-ins) and blow downs (zoom-outs) of measures with positive finite density. We say a Radon measure $\mu$ in $\mathbf{R}^{d}$ is uniformly distributed if there exists a positive function $\phi: \mathbf{R}_{+} \rightarrow \mathbf{R}_{+}$, called its distribution function, such that

$$
\mu(B(x, r))=\phi(r) \text {, for all } x \in \operatorname{supp} \mu \text {, for all } r>0 .
$$

An example of note is when the function $\phi$ is $c r^{n}$ for some $c>0, n \leq d$. These are called $n$-uniform measures and appear in many different contexts from geometric measure theory to harmonic analysis and PDE's (for instance in [DKT] and [PTT]).

The geometry of the supports of uniformly distributed measures is not very well understood. Let us start by stating some known facts. As a direct consequence of Preiss' theorem, we can deduce that the support of an $n$-uniform measure is countably $n$-rectifiable. In fact, the same can be said of uniformly distributed measures. Indeed, Preiss proved in $[\mathrm{P}]$ that uniformly distributed measures "look like" $n$-uniform measures on small and on large scales. Their $n$-rectifiability can easily be deduced from that fact.

One might expect much more regularity than rectifiability, given the fact that the property of being uniformly distributed is a global one (i.e. it is a property for

https://doi.org/10.5186/aasfm.2019.4422

2010 Mathematics Subject Classification: Primary 28A33, 49Q15. graphs.

Key words: Uniformly distributed measures, uniform rectifiability, big pieces of Lipschitz

The author was partially supported by NSF RTG 0838212, DMS-1361823 and DMS-0856687. 
all $r>0$ ). This turns out to be the case. For $n$-uniform measures, a classification is available in some cases. In [P], Preiss provides a classification of the cases $n=1,2$ in $\mathbf{R}^{d}$ for any $d$. In these cases, $\mu$ is Hausdorff measure restricted to a line or a plane respectively. In $[\mathrm{KoP}]$, Kowalski and Preiss proved that $\mu$ is $(d-1)$-uniform in $\mathbf{R}^{d}$ if and only if $\mu=\mathcal{H}^{d-1}\llcorner V$ where $V$ is a $(d-1)$-plane, or $d \geq 4$ and there exists an orthonormal system of coordinates in which $\mu=\mathcal{H}^{d-1}\llcorner(C \times W)$ where $W$ is a $(d-4)$-plane and $C$ is the KP-cone (Kowalski-Preiss cone)

$$
C=\left\{\left(x_{1}, x_{2}, x_{3}, x_{4}\right) ; x_{4}^{2}=x_{1}^{2}+x_{2}^{2}+x_{3}^{2}\right\} .
$$

The classification for $n \geq 3$ and codimension $\geq 2$ remains an open question.

On the other hand, in [KiP], Kirchheim and Preiss proved that the support of a uniformly distributed measure is an analytic variety, that is the intersection of zero sets of analytic functions. More precisely:

Theorem 1.1. [KiP] Let $\mu$ be a uniformly distributed measure over $\mathbf{R}^{d}$ and let $u \in \Sigma$ where $\Sigma=\operatorname{supp} \mu$. For every $x \in \mathbf{R}^{d}$ and $s>0$, let

$$
F(x, s)=\int_{\mathbf{R}^{d}} e^{-s|z-x|^{2}}-e^{-s|z-u|^{2}} d \mu(z)
$$

Then:

- $F(x, s)$ is well-defined and finite for any $x \in \mathbf{R}^{d}$ and any $s>0$; moreover its definition is independent of the choice of $u \in \Sigma$

- $\Sigma=\bigcap_{s>0}\left\{x \in \mathbf{R}^{d} ; F(x, s)=0\right\}$

It is a known fact that an analytic variety of dimension $n$ is a finite union of analytic $n$-submanifolds up to a set of $\mathcal{H}^{n}$-measure 0 . This confirms the expectation of regularity but has the disadvantage of not providing any quantitative information on the regularity of the support.

Let us now turn to uniform rectifiability. This notion was introduced by David and Semmes (see for example [DS2]). It is a quantitative version of the notion of $n$-rectifiability. One possible definition of it is the following.

Let $\mu$ be a Radon measure in $\mathbf{R}^{d}$, and $\Sigma$ its support, $0 \in \Sigma$. We say that an Ahlfors $n$-regular measure $\mu$ has big pieces of Lipschitz graphs (BPLG) if there exist constants $\theta$ and $M$ so that, for each $x \in \Sigma$ and $R>0$, there is a Lipschitz function $g$ from $\mathbf{R}^{n}$ to $\mathbf{R}^{d-n}$ such that $g$ has Lipschitz norm not exceeding $M$ and such that its graph $\Gamma$ (up to rotation) satisfies

$$
\mu(B(x, R) \cap \Gamma) \geq \theta R^{n} .
$$

We say that $\mu$ has BPLG locally if given $K$ a compact set of $\mathbf{R}^{d}$ then for every $x \in \Sigma \cap K$ and every $0<R<\operatorname{diam}(K)$, there is a Lipschitz function $g$ from $\mathbf{R}^{n}$ to $\mathbf{R}^{d-n}$ such that $g$ has Lipschitz norm not exceeding $M$ and such that its graph $\Gamma$ (up to rotation) satisfies

$$
\mu(B(x, R) \cap \Gamma) \geq \theta R^{n} .
$$

In [T], Tolsa proved that $n$-uniform measures have BPLG.

Theorem 1.2. [T] Let $\mu$ be an $n$-uniform measure in $\mathbf{R}^{d}$. Then $\mu$ has big pieces of Lipschitz graphs.

Since uniformly distributed measures "look like" $n$-uniform measures on small scales one might expect this result to hold locally for uniformly distributed measures. In this paper, we will prove that this is indeed the case. Namely, we will prove the following theorem: 
Theorem 1.3. Let $\mu$ be a uniformly distributed measure in $\mathbf{R}^{d}$. Then $\mu$ has big pieces of Lipschitz graph locally.

The proof is analogous to Tolsa's proof of Theorem 1.2. To apply the techniques that the author introduced in $[\mathrm{T}]$, one needs to use the fact that uniformly distributed measures locally behave like $n$-uniform measures and are radially invariant. These two properties allow us to obtain estimates on the Riesz transforms and to prove that every ball in $\Sigma$ the support of $\mu$ contains a relatively large ball that is flat.

The second step consists in proving that flatness is stable for uniformly distributed measures. In other words, if the support is flat at small enough scale it will be flat at all smaller scales. The fact that uniformly rectifiable measures have $n$-uniform pseudo-tangents is the key idea allowing us to generalize the stability of flatness for $n$-uniform measures to uniformly distributed measures.

\section{Preliminaries}

Let us first define the support of a measure.

Definition 2.1. Let $\mu$ be a measure in $\mathbf{R}^{d}$. We define the support of $\mu$ to be

$$
\operatorname{supp}(\mu)=\left\{x \in \mathbf{R}^{d} ; \mu(B(x, r))>0 \text {, for all } r>0\right\} .
$$

Note that the support of a measure is a closed subset of $\mathbf{R}^{d}$.

We start with some facts about uniformly distributed measures. The first is a theorem by Preiss describing the behavior of uniformly distributed measures at small and large scales.

Theorem 2.2. [P, Theorem 3.11] Suppose $\mu$ is uniformly distributed in $\mathbf{R}^{d}$, and let $\phi$ be its distribution function. Then there exist integers $n$ and $p$ such that

$$
\lim _{r \rightarrow 0} \frac{\phi(r)}{r^{n}} \text { and } \lim _{r \rightarrow \infty} \frac{\phi(r)}{r^{p}} \text { both exist, are positive and finite. }
$$

We denote $n$ and $p$ by

$$
n=\operatorname{dim}_{0} \mu \text { and } p=\operatorname{dim}_{\infty} \mu .
$$

We can deduce the following useful corollary about the growth of $\mu$ at small scales from this theorem.

Corollary 2.3. Suppose $\mu$ is a uniformly distributed measure with $\operatorname{dim}_{0} \mu=n$ and $\operatorname{dim}_{\infty} \mu=p$, and $\phi$ the function associated to $\mu$. Let $R \in \mathbf{R}_{+}$. There exists $C \in \mathbf{R}_{+}$depending on $R$ such that for all $r \leq R$, the following holds:

$$
C^{-1} r^{n} \leq \phi(r) \leq C r^{n}
$$

Proof. According to Theorem 2.2 there exist $r_{0}$ and $r_{\infty}$ such that

$$
\begin{array}{ll}
\mu(B(x, r)) \sim r^{n}, & x \in \Sigma, r \leq r_{0}, \\
\mu(B(x, r)) \sim r^{p}, & x \in \Sigma, r \geq r_{\infty}
\end{array}
$$

If $R \leq r_{0}$, the statement follows with a $C$ not depending on $R$. First, assume $r_{0} \leq R \leq r_{\infty}$ and take $r$ such that $r_{0} \leq r \leq R$. Then

$$
\frac{r_{0}{ }^{n}}{R^{n}} r^{n} \lesssim \phi\left(r_{0}\right) \leq \phi(r) \leq \phi\left(r_{\infty}\right) \lesssim \frac{r_{\infty}^{p}}{r_{0}{ }^{n}} r^{n}
$$

Now assume $R \geq r_{\infty}$ and let $r \leq R$. If $r_{0} \leq r \leq r_{\infty}$, then

$$
\frac{r_{0}{ }^{n}}{r_{\infty}{ }^{n}} r^{n} \leq r_{0}{ }^{n} \lesssim \phi\left(r_{0}\right) \leq \phi(r) \leq \phi\left(r_{\infty}\right) \lesssim r_{\infty}{ }^{p} \leq \frac{r_{\infty}^{p}}{r_{0}{ }^{n}} r^{n} .
$$


Finally, suppose $r_{\infty} \leq r \leq R$. Then

$$
\frac{r_{\infty}^{p}}{R^{n}} r^{n} \leq r_{\infty}^{p} \lesssim \phi(r) \lesssim r^{p} \leq \frac{R^{p}}{r_{\infty}{ }^{n}} r^{n}
$$

Another theorem in $[\mathrm{KiP}]$ states that uniformly distributed measures don't grow too fast.

Theorem 2.4. [KiP, Lemma 1.1] Let $\mu$ be a uniformly distributed measure over $\mathbf{R}^{d}, x \in \mathbf{R}^{d}, 0<s<r<\infty$ and $\phi$ its distribution function. Then $\mu(B(x, r)) \leq$ $5^{d}\left(\frac{r}{s}\right)^{d} \phi(s)$.

Another interesting feature of uniformly distributed measures is that radial functions integrate nicely against them.

Theorem 2.5. [M] Let $\mu$ be a uniformly distributed measure on $\mathbf{R}^{d}$ and $f$ be a non-negative Borel function on $\mathbf{R}_{+}$. For all $z, y \in \operatorname{supp}(\mu)$, we have

$$
\int f(|x-z|) d \mu(x)=\int f(|x-y|) d \mu(x) .
$$

Next, we introduce the following beta numbers initially introduced by Jones. They quantify how "flat" (or far from a plane) the support of a measure is.

Definition 2.6. Let $\mu$ be a Radon measure in $\mathbf{R}^{d}$, and $\Sigma$ its support.

- We define Jones' $\beta_{\mu}^{n}$ number of $B$ to be

$$
\beta_{\mu}^{n}(B)=\inf _{L} \sup _{x \in \Sigma \cap B} \frac{\operatorname{dist}(x, L)}{r},
$$

where $B$ is a ball in $\mathbf{R}^{d}$, and the infimum is taken over all $n$-planes.

- We define the bilateral beta number $b \beta_{\mu}^{n}$ of $B$ to be

$$
b \beta_{\mu}^{n}(B)=\inf _{L}\left(\sup _{x \in \Sigma \cap B} \frac{\operatorname{dist}(x, L)}{r}+\sup _{p \in L \cap B} \frac{\operatorname{dist}(p, \Sigma)}{r}\right),
$$

where the infimum is taken over all $n$ planes in $\mathbf{R}^{d}$. We will drop the $n$ superscript and $\mu$ subscript when there is no ambiguity.

- We say $\mu$ is $n$-flat if there exists an $n$-dimensional plane $V$ in $\mathbf{R}^{d}$ and a constant $c>0$ such that $\mu=c \mathcal{H}^{n}\llcorner V$.

Let us define doubling measures.

Definition 2.7. Let $\mu$ be a measure in $\mathbf{R}^{d}$. We say $\mu$ is a doubling measure if there exists $C>0$ such that

$$
\mu(B(x, 2 r)) \leq C \mu(B(x, r)) \text {, for all } x \in \operatorname{supp}(\mu) \text {, for all } r>0 .
$$

The smallest such $C$ is called the doubling constant of $\mu$.

The two following lemmas relate the weak convergence of a sequence of doubling measures to the convergence of their supports as sets in $\mathbf{R}^{d}$. They are analogues of Lemmas 2.2 and 2.3 from [T].

Lemma 2.8. Let $\mu_{j}, \mu$ be doubling Radon measures, all having their doubling constants bounded by the same positive $C>0$. Let $\Sigma_{j}, \Sigma$ be the supports of $\mu_{j}$ and $\mu$ respectively, and $\bar{B}$ a closed ball in $\mathbf{R}^{d}$ such that $\bar{B} \cap \Sigma \neq \emptyset$, and $\bar{B} \cap \Sigma_{j} \neq \emptyset$ for all $j$. If $\mu_{j}$ converges weakly to $\mu\left(\mu_{j} \rightarrow \mu\right)$, then $d_{\bar{B}, 2 \bar{B}}\left(\Sigma_{j}, \Sigma\right)$ converges to 0 , where

$$
d_{\bar{B}, 2 \bar{B}}(U, V)=\sup _{x \in U \cap \bar{B}} \operatorname{dist}(x, V \cap 2 \bar{B})+\sup _{x \in V \cap \bar{B}} \operatorname{dist}(x, U \cap 2 \bar{B}) .
$$


Proof. We first prove that $\sup _{p \in \Sigma_{j} \cap \bar{B}} \operatorname{dist}(p, \Sigma \cap 2 \bar{B}) \rightarrow 0$. Suppose not. Then, without loss of generality there exists $\epsilon>0, p_{j} \in \Sigma_{j} \cap B$, for $j>0$, such that

$$
B\left(p_{j}, 2 \epsilon\right) \cap \Sigma \cap 2 \bar{B}=\emptyset \text {. }
$$

In particular, $\mu\left(B\left(p_{j}, 2 \epsilon\right)\right)=0$. Let $\chi_{j}, \tilde{\chi}$ be functions compactly supported in $4 \bar{B}$ such that $\chi_{B\left(p_{j}, \epsilon\right)} \leq \chi_{j} \leq \chi_{B\left(p_{j}, 2 \epsilon\right)}$, and $\chi_{\bar{B}} \leq \tilde{\chi} \leq \chi_{3 \bar{B}}$. There exists $k_{j} \geq 0$ such that

$$
2 \bar{B} \subset B\left(p_{j}, 2^{k_{j}} \epsilon\right)
$$

In particular, since $p_{j} \in \bar{B}$ is closed,

$$
k_{j} \leq \frac{\log (2 r(B))-\log (\epsilon)}{\log (2)} \leq K,
$$

where $K$ does not depend on $j$. Since $\mu_{j}$ are all doubling, we have:

$$
C^{-K} \mu_{j}(2 \bar{B}) \leq \mu_{j}\left(B\left(p_{j}, \epsilon\right)\right) \leq \int \chi_{j} d \mu_{j}
$$

On one hand, $\mu_{j}(2 \bar{B}) \geq \int \tilde{\chi} d \mu_{j}$ and $\int \tilde{\chi} d \mu_{j} \rightarrow \int \tilde{\chi} d \mu>0$ imply that liminf $\int \chi_{j} d \mu_{j}>$ 0 . On the other hand, since $\int \chi_{j} d \mu_{j} \leq \int \tilde{\chi} d\left(\mu-\mu_{j}\right)+\mu\left(B\left(p_{j}, 2 \epsilon\right)\right)$, then $\int \chi_{j} d \mu_{j} \rightarrow 0$ as $j \rightarrow \infty$, yielding a contradiction.

We now prove that $\sup _{p \in \Sigma \cap \bar{B}} \operatorname{dist}\left(p, \Sigma_{j} \cap 2 \bar{B}\right) \rightarrow 0$. Suppose not. Then there exists $\epsilon>0$, and, without loss of generality, points $x_{j} \in \Sigma \cap \bar{B}$ such that: $B\left(x_{j}, 2 \epsilon\right) \cap$ $\Sigma_{j} \cap 2 \bar{B}=\emptyset$. In particular, $\mu_{j}\left(B\left(x_{j}, 2 \epsilon\right)\right)=0$. Passing to a subsequence, we can assume that $x_{j} \rightarrow x, x \in \Sigma \cap \bar{B}$ since $\Sigma \cap \bar{B}$. So there exists $N$ such that, when $j>N,\left|x-x_{j}\right|<\epsilon$. Consequently, $B(x, \epsilon) \subset B\left(x_{j}, 2 \epsilon\right)$ and $\mu_{j}(B(x, \epsilon))=0$. Let $\phi$ be a function compactly supported in $4 \bar{B}$ such that $\chi_{B\left(x, \frac{\epsilon}{5}\right)} \leq \phi \leq \chi_{B(x, \epsilon)}$. Then, on one hand, we have $\int \phi d \mu_{j}=0$, implying that $\int \phi d \mu=\lim \int \phi d \mu_{j}=0$. On the other hand, $\int \phi d \mu \geq \mu\left(B\left(x, \frac{\epsilon}{5}\right)\right)>0$, yielding a contradiction.

Theorem 2.9. Let $\mu_{j}, \mu$ be doubling measures, with the same doubling constant $c, B$ a ball such that $\mu_{j} \rightarrow \mu, \Sigma_{j} \cap B \neq \emptyset, \Sigma \cap B \neq \emptyset$. Let $0<n \leq d$. Then

$$
\begin{aligned}
& \frac{1}{2} \lim \sup \beta_{\mu_{j}}^{n}\left(\frac{1}{2} B\right) \leq \beta_{\mu}^{n}(B) \leq 2 \liminf \beta_{\mu_{j}}^{n}(2 B), \\
& \frac{1}{2} \lim \sup b \beta_{\mu_{j}}^{n}\left(\frac{1}{2} B\right) \leq b \beta_{\mu}^{n}(B) \leq 2 \liminf b \beta_{\mu_{j}}^{n}(2 B) .
\end{aligned}
$$

Proof. The proof is an easy consequence of Lemma 2.8. We prove that $\beta_{\mu}^{n}(B) \leq$ $2 \liminf \beta_{\mu_{j}}^{n}(2 B)$ as an example. Take any $x \in \Sigma_{j} \cap B$. Let $y \in \Sigma \cap 2 B$ be such that $|x-y|=\operatorname{dist}(x, \Sigma \cap 2 B)$. Pick any $n$-plane $L$. Then $\operatorname{dist}(x, L) \leq \operatorname{dist}(x, \Sigma \cap$ $2 B)+\operatorname{dist}(y, L)$, implying that $\inf _{L} \sup _{x \in \Sigma_{j} \cap B} \operatorname{dist}(x, L) \leq \sup _{x \in \Sigma_{j} \cap B} \operatorname{dist}(x, \Sigma \cap 2 B)+$ $\inf _{L} \sup _{y \in \Sigma \cap 2 B} \operatorname{dist}(y, L)$. Therefore, $\beta_{\mu}^{n}(B) \leq 2 \beta_{\mu_{j}}^{n}(2 B)$.

To describe the local geometry of a measure, we study objects called its tangents and pseudo-tangents.

Definition 2.10. Let $\mu$ be a Radon measure on $\mathbf{R}^{d}$.

- We say that $\nu$ is a tangent measure of $\mu$ at a point $a \in \mathbf{R}^{d}$ if $\nu$ is a non-zero Radon measure on $\mathbf{R}^{d}$, and if there exist sequences $\left(r_{i}\right)$ and $\left(c_{i}\right)$ of positive numbers such that $r_{i} \rightarrow 0$ and $c_{i} T_{a, r_{i}} \sharp \mu \rightarrow \nu$, as $i \rightarrow \infty$. Here, $T_{a, r_{i}} \sharp \mu$ is the push-forward of $\mu$ under the bijection $T_{a, r}(x)=\frac{x-a}{r}$. 
- Let $\Sigma$ denote the support of the measure $\mu$. We say that $\mu$ is $n$-uniform if there exists $c>0$ such that for all $x \in \Sigma$, for all $r>0$, the following holds:

$$
\mu(B(x, r))=c r^{n} .
$$

In [P, Theorem 3.11], Preiss showed that if $\mu$ is an uniformly distributed measure, there exists a unique $n$-uniform measure $\lambda$ such that:

$$
r^{-n} T_{x, r} \sharp \mu \rightarrow \lambda, \text { as } r \rightarrow \infty,
$$

for all $x \in \mathbf{R}^{d}$. $\lambda$ is called the tangent measure of $\mu$ at $\infty$.

A remarkable fact about this measure $\lambda$ is the following "connectedness at $\infty$ " for the cone of uniform measures. The following is a version of this result formulated by Tolsa in $[\mathrm{T}]$.

Theorem 2.11. [P] Suppose $\mu$ is a uniformly distributed measure in $\mathbf{R}^{d}, \lambda$ its tangent at $\infty$.

- If $n=1,2$, then $\mu$ is flat.

- If $n \geq 3$, there exists a constant $\tau_{0}$ depending only on $n$ and $d$ such that, if $\lambda$ satisfies the following:

$$
\beta_{\lambda}^{n}(B(0,1)) \leq \tau_{0}
$$

then $\mu$ is $n$-flat.

Let us define the notion of asymptotically optimally doubling measures.

Definition 2.12. If $x \in \Sigma, r>0$ and $t \in(0,1]$, define the quantity

$$
R_{t}(x, r)=\frac{\mu(B(x, t r))}{\mu(B(x, r))}-t^{n} .
$$

We say $\mu$ is asymptotically optimally doubling if for each compact set $K \subset \Sigma, x \in K$, and $t \in\left[\frac{1}{2}, 1\right]$

$$
\lim _{r \rightarrow 0^{+}} \sup _{x \in K}\left|R_{t}(x, r)\right|=0 .
$$

We define Ahlfors regular and locally Ahlfors regular measures.

Definition 2.13. Let $\mu$ be a Radon measure in $\mathbf{R}^{d}$, and $\Sigma$ its support.

- We say $\mu$ is Ahlfors $n$-regular, $0<n \leq d$ if there exists a constant $c_{1}$ such that:

$$
c_{1}^{-1} r^{n} \leq \mu(B(x, r)) \leq c_{1} r^{n}, \text { for all } x \in \Sigma, r>0 .
$$

- We say $\mu$ is locally Ahlfors $n$-regular if for all $K$ compact, there exist constants $c_{K}>0$ and $r_{K}$ such that, for all $x \in \Sigma \cap K, 0<r \leq r_{K}$,

$$
c_{K}^{-1} r^{n} \leq \mu(B(x, r)) \leq c_{K} r^{n} .
$$

The notion of uniform rectifiability was introduced by David and Semmes in [DS2]. It is a quantitative version of the notion of $n$-rectifiability.

Definition 2.14. Let $\mu$ be a Radon measure in $\mathbf{R}^{d}$, and $\Sigma$ its support.

- We say $\mu$ is uniformly $n$-rectifiable if it is Ahlfors $n$-regular, and there exist constants $\theta$ and $M$ so that, for each $x \in \Sigma$ and $R>0$, there is a Lipschitz mapping $g$ from $\mathbf{R}^{n}$ to $\mathbf{R}^{d}$ such that $g$ has Lipschitz norm not exceeding $M$ and such that

$$
\mu\left(B(x, R) \cap g\left(\mathbf{R}^{n}\right)\right) \geq \theta R^{n} .
$$

We say $\Sigma$ has big pieces of Lipschitz images (BPLI). 
- We say $\mu$ is locally uniformly $n$-rectifiable if it is locally Ahlfors $n$-regular, and for every compact set $K$, there exist constants $R_{K}, \theta_{K}$ and $M_{K}$ so that, for each $x \in \Sigma \cap K$ and $0<R \leq R_{K}$, there is a Lipschitz mapping $g$ from $\mathbf{R}^{n}$ to $\mathbf{R}^{d}$ such that $g$ has Lipschitz norm not exceeding $M_{K}$ and such that

$$
\mu\left(B(x, R) \cap g\left(\mathbf{R}^{n}\right)\right) \geq \theta_{K} R^{n} .
$$

Our final definition is of the Riesz transforms of a measure.

Definition 2.15. Let $\mu$ be a Radon measure in $\mathbf{R}^{d}$. The Riesz transform of $\mu$ for $z_{0} \in \operatorname{supp}(\mu), 0<r<s$ is defined as

$$
R_{r, s} \mu\left(z_{0}\right)=\int_{r \leq\left|z_{0}-y\right| \leq s} \frac{z_{0}-y}{\left|z_{0}-y\right|^{n+1}} d \mu(y)
$$

In [T], the following estimate on the Riesz transform was an essential tool for Tolsa's proof of the uniform rectifiability of $n$-uniform measures.

Lemma 2.16. [T] Let $\mu$ be a Radon measure, $\Sigma=\operatorname{supp}(\mu), n \leq d$. Let $B$ be a ball centered in $\Sigma$ and $r(B)$ its radius. Suppose that there exist constants $\kappa, c_{1}$ such that

$$
c_{1}^{-1} r^{n} \leq \mu(B(x, r)) \leq c_{1} r^{n}, \text { for } x \in B \cap \Sigma, \kappa r(B) \leq r \leq r(B) .
$$

Moreover, suppose that for some $\epsilon>0$, we have

$$
\beta_{\mu}^{d-1}(B(x, r)) \geq \epsilon, \text { for } x \in B \cap \Sigma, \kappa r(B) \leq r \leq r(B) .
$$

Then, for any $M>0$, there exists $\kappa_{0}\left(\kappa_{0}=\kappa\left(M, \epsilon, c_{1}\right)\right)$, such that if $\kappa \leq \kappa_{0}$, then there exists $r, \kappa r(B) \leq r \leq r(B)$, and points $x, z_{0} \in B \cap \Sigma$, with $\left|x-z_{0}\right| \leq \kappa r(B)$ satisfying

$$
\left|\frac{x-z_{0}}{\kappa r(B)} \cdot R_{\kappa r(B), r} \mu\left(z_{0}\right)\right| \geq M
$$

Finally, we state the following theorem from [DT] which will be used in the final step of the proof.

Theorem 2.17. [DT] Let $E$ be a set that is locally Ahlfors-regular and that satisfies for $\epsilon$ small enough, $b \beta_{\mu}(B(x, r)) \leq \epsilon$, for every $x \in E$ and every $0 \leq r \leq 1$ where,$\mu=\mathcal{H}^{n}\llcorner E$. Then there exist constants $\theta>0$ and $M>0$ depending only on $n, d$ and the Ahlfors regularity constant such that for $x \in E$ and $0<r \leq 1$, we can find a $n$-plane $P$ and an $M$-Lipschitz function $F: P \rightarrow P^{\perp}$ such that

$$
\mu\left(\Gamma_{F} \cap B(x, r)\right) \geq \theta r^{d},
$$

where $\Gamma_{F}$ denotes the graph of $F$.

\section{Existence of big flat balls for uniformly distributed measures}

We start by proving that the Riesz transform of a uniformly distributed measure is locally bounded. The two following lemmas are local analogues to Lemmas 3.1 and 3.4 in [T] for uniformly distributed measures. Their proofs follow closely the proofs of their counterparts in $[\mathrm{T}]$.

Lemma 3.1. Let $\mu$ be a uniformly distributed measure, $\operatorname{dim}_{0}(\mu)=n, R>0$. Let $z_{0} \in \Sigma, 0<r \leq R$. Then we have

$$
\left|\frac{x-z_{0}}{r} \cdot R_{r, s} \mu\left(z_{0}\right)\right| \leq c, \text { for all } r<s \leq \frac{R}{2} \text {, and for all } x \in B\left(z_{0}, r\right)
$$


where $c$ depends only on $R$.

Proof. Without loss of generality, assume $z_{0}=0$. For $r, s$ fixed, $0<r<s \leq \frac{R}{2}$, define the function $\psi: \mathbf{R} \rightarrow \mathbf{R}$ to be a compactly supported $C^{\infty}$ function with the following properties:

$$
\psi(t)= \begin{cases}0 & \text { if }|t| \geq 2 s \text { or }|t| \leq \frac{r}{2} \\ \frac{1}{t^{n}} & \text { if } r \leq|t| \leq s\end{cases}
$$

and

$$
|\psi(t)| \leq c \min \left(\frac{1}{r^{n}}, \frac{1}{t^{n}}\right) \text { for all } t \in \mathbf{R} .
$$

We also require that

$$
\left|\psi^{\prime}(t)\right| \leq c \min \left(\frac{1}{(3 r)^{n+1}}, \frac{1}{t^{n+1}}\right) \text { for all } t \in \mathbf{R} .
$$

We define real-valued functions $\rho$ and $\Psi$ respectively from $\mathbf{R}$ and $\mathbf{R}^{d}$ as follows:

$$
\rho(u)=-\int_{u}^{\infty} \psi(t) d t, \quad u \in \mathbf{R},
$$

and

$$
\Psi(y)=\rho(|y|), \quad y \in \mathbf{R}^{d}
$$

Since $\mu$ is uniformly distributed and $\Psi$ is radial, for all $x \in B(0, r) \cap \operatorname{supp}(\mu)$, we have by Theorem 2.5

$$
\int \Psi(x-y) d \mu(y)-\int \Psi(y) d \mu(y)=0 .
$$

On the other hand, Taylor's formula gives

$$
\Psi(x-y)-\Psi(-y)=x \cdot \nabla \Psi(-y)+\frac{1}{2} x^{T} \cdot \nabla^{2} \Psi\left(\xi_{x, y}\right) \cdot x,
$$

where $\xi_{x, y} \in[x-y,-y] \subset \mathbf{R}^{d}$. Note that $\nabla \Psi(z)=\psi(|z|) \cdot \frac{z}{|z|}$ implying that

$$
-R_{r, s} \mu(0)=\int_{r \leq|y| \leq s} \nabla \Psi(-y) d \mu(y)
$$

since $\psi(|z|)=\frac{1}{|z|^{n}}$ when $|z| \in(r, s)$. Combining (3.3),(3.4) and (3.5), we get

$$
\begin{aligned}
& x \cdot \int_{|y| \leq r} \nabla \Psi(-y) d \mu(y)-x \cdot R_{r, s} \mu(0)+x \cdot \int_{|y|>s} \nabla \Psi(-y) d \mu(y) \\
& +\frac{1}{2} x^{T} \cdot\left(\int \nabla^{2} \Psi\left(\xi_{x, y}\right) d \mu(y)\right) \cdot x=0 .
\end{aligned}
$$

This gives

$$
(3.6) x \cdot R_{r, s} \mu(0)=x \cdot \int_{\{|y| \leq r\} \cup\{|y|>s\}} \nabla \Psi(-y) d \mu(y)+\frac{1}{2} x^{T} \cdot\left(\int \nabla^{2} \Psi\left(\xi_{x, y}\right) d \mu(y)\right) \cdot x .
$$

Let us estimate the right hand-side of (3.6). Using the inequalities (3.1) and (3.2) and Corollary 2.3, we get the following estimates on the first term in (3.6):

$$
\left|\int_{|y| \leq r} \nabla \Psi(-y) d \mu(y)\right| \leq \frac{c}{r^{n}} \mu(B(0, r)) \leq C \text { since } r \leq R
$$


and since $2 s \leq R$

$$
\left|\int_{|y|>s} \nabla \Psi(-y) d \mu(y)\right|=\left|\int_{s<|y| \leq 2 s} \nabla \Psi(-y) d \mu(y)\right| \leq \frac{1}{s^{n}} \mu(B(0,2 s)) \leq C 2^{n},
$$

where $C$ depends on $R$. Let us now estimate the second order derivative of $\Psi$ using the fact that

$$
\left|\nabla^{2} \Psi\left(\xi_{x, y}\right)\right| \leq c \min \left(\frac{1}{3 r^{n+1}}, \frac{1}{\left|\xi_{x, y}\right|^{n+1}}\right) .
$$

More specfically, we show that

$$
\int\left|\nabla^{2} \Psi\left(\xi_{x, y}\right)\right| d \mu(y) \leqslant \frac{1}{r}
$$

If $|y| \leq 2 r$, we get $\left|\nabla^{2} \Psi\left(\xi_{x, y}\right)\right| \leq \frac{C}{r^{n+1}}$. If $|y|>2 r$, then $\frac{|y|}{2} \leq|x-y| \leq 2|y|$ implies that $\left|\xi_{x, y}\right| \sim|y|$ and hence $\left|\nabla^{2} \Psi\left(\xi_{x, y}\right)\right| \leq \frac{C}{|y|^{n+1}}$. Therefore, since $\mu$ is uniformly distributed, and $\psi$ compactly supported in $[-R, R]$, we get

$$
\begin{aligned}
\int\left|\nabla^{2} \Psi\left(\xi_{x, y}\right)\right| d \mu(y) & \leq c \int_{|y| \leq 2 r} \frac{1}{r^{n+1}} d \mu(y)+c \int_{2 r<|y| \leq R} \frac{1}{|y|^{n+1}} d \mu(y), \\
& \leq \frac{c}{r}+c \int_{2 r<|y| \leq R} \frac{1}{|y|^{n+1}} d \mu(y), \quad \text { since } 2 r \leq R
\end{aligned}
$$

We claim that

$$
\int_{2 r<|y| \leq R} \frac{1}{|y|^{n+1}} d \mu(y) \leq \frac{c}{r}
$$

Indeed,

$$
\begin{aligned}
\int_{2 r<|y| \leq R} \frac{1}{|y|^{n+1}} d \mu(y) & =\int_{\frac{1}{R^{n+1}}}^{\frac{1}{(2 r)^{n+1}}} \mu\left(\left\{y:|y|<\frac{1}{t^{\frac{1}{(n+1)}}}\right\}\right) d t \\
& =\int_{\frac{1}{R^{n+1}}}^{\frac{1}{(2 r)^{n+1}}} \mu\left(B\left(0, \frac{1}{t^{(n+1)}}\right)\right) d t \\
& \leq c \int_{\frac{1}{R^{n+1}}}^{\frac{1}{(2 r)^{n+1}}} \frac{1}{t^{\frac{n}{(n+1)}}} d t=c\left(\frac{1}{2 r}-\frac{1}{R}\right) \leq \frac{c}{2 r}
\end{aligned}
$$

This gives

$$
\left|x \cdot R_{r, s} \mu(0)\right| \leq c|x|+c \frac{|x|^{2}}{r} \leq c r
$$

since $|x| \leq r$.

Lemma 3.2. Let $\mu$ be a uniformly distributed measure, $n=\operatorname{dim}_{0} \mu, n<m \leq d$, $\epsilon>0$. Let $K$ be a compact set, $R=\operatorname{diam}(K)$. There exist constants $\delta, \tau$ depending only on $\epsilon, K, n$ and $d$ such that if $B$ is a ball centered in $\Sigma \cap K$, with $r(B) \leq R$ and $\beta_{\mu}^{m}(B) \leq \delta$, then there exists a ball $B^{\prime}, B^{\prime} \subset B$, centered in $\Sigma$ such that $\beta_{\mu}^{m-1}\left(B^{\prime}\right) \leq \epsilon$ and $r\left(B^{\prime}\right) \geq \tau \cdot r(B)$ where $r(B)$ denotes the radius of $B$.

Proof. Let $L$ be a best approximating $m$-plane for $\beta_{\mu}^{m}(B)$. In particular, for any $z \in \Sigma \cap B$,

$$
|z-\pi(z)|<\delta r(B)
$$


assuming that $\beta_{\mu}^{m}(B) \leq \delta$ for a $\delta$ to be chosen later. Denote $\pi_{L}$, the orthogonal projection onto $L$, by $\pi$ and define the measure $\nu$ on $L$ to be

$$
\nu(A)=\mu\left(\pi^{-1}(A) \cap B\right) \text { for } A \subset L .
$$

Let $x \in \frac{1}{2} B \cap \widetilde{\Sigma}$, where $\widetilde{\Sigma}=\operatorname{supp} \nu$. On one hand, denoting by $B_{L}(x, r)$ the ball in $L$ i.e. $B(x, r) \cap L$, we have $B(x, r) \subset \pi^{-1}\left(B_{L}(x, r)\right) \cap B$ when $r \leq \frac{r(B)}{2}$, and hence

$$
c^{-1} r^{n} \leq \mu(B(x, r)) \leq \nu\left(B_{L}(x, r)\right) .
$$

On the other hand, if $J$ is the maximal number of disjoint balls of radius $r$ that can be contained in $\pi^{-1}\left(B_{L}(x, r)\right) \cap 2 B$, then we claim that

$$
J \leq \omega_{m}(2 r)^{m-d} 2 r(B)^{d-m} .
$$

Indeed, assuming that $L$ is the $m$-plane $\left\{\left(y_{1}, \ldots, y_{d}\right) ; y_{m+1}=\ldots=y_{d}=0\right\}$, the union $E$ of these $J$ balls is included in the cylinder

$$
C=\left\{y ;\left(y_{1}, \ldots, y_{m}\right) \in B_{L}(x, r), y_{j} \in(-2 r(B), 2 r(B)), j>m\right\} .
$$

Thus

$$
J \cdot \mathcal{L}^{d}(B(0, r)) \leq \mathcal{L}^{d}(\mathcal{C})=\omega_{m} r^{m} 2 r(B)^{d-m} .
$$

Let $\left\{B_{i}\right\}$ be a disjoint collection of balls of radius $r$ such that $B_{i} \subset \pi^{-1}\left(B_{L}(x, r)\right) \cap$ $2 B \subset \bigcup 5 B_{i}$, obtained by a Vitali covering. In particular, by the above argument, there are at most $J$ of them. Then, if $\delta r(B) \leq r \leq r(B)$, where $\delta$ is to be chosen later, we have

$$
\nu\left(B_{L}(x, r)\right) \leq \mu\left(\bigcup 5 B_{i}\right) \leq J \cdot c \cdot(r)^{n} \leq(2 r)^{m-d} \omega_{m} r(B)^{d-m}(2 r)^{n} \leq \frac{c}{\delta^{d-m}} r^{n} .
$$

Hence, letting $C=\frac{c}{\delta^{d-m}}$, we have

$$
C^{-1} r^{n} \leq \nu(B(x, r)) \leq C r^{n}, \quad \text { when } x \in \frac{1}{2} B \cap \widetilde{\Sigma}, \delta r(B) \leq r \leq r(B) .
$$

We first claim that for all $z_{0} \in \frac{1}{2} B \cap \widetilde{\Sigma}$, and $r_{0}, r$ with $\delta^{\frac{1}{2}} r(B) \leq r_{0} \leq r \leq r(B)$, if $\delta$ is small enough, then

$$
\left|\frac{x-z_{0}}{r_{0}} R_{r_{0}, r} \nu\left(z_{0}\right)\right| \leq c, \quad \text { for } x \in \widetilde{\Sigma} \cap B\left(z_{0}, r_{0}\right) .
$$

Let us finish the proof of the lemma before proving (3.8). Choose $\epsilon>0$. Let $\kappa_{0}=\kappa_{0}(\epsilon)$ be as in Lemma 2.16. Let $\delta$ be small enough for (3.8) to hold, $\delta^{\frac{1}{2}} \leq \kappa_{0}$ and $\delta^{\frac{1}{2}} \leq \frac{\epsilon}{2}$. Identify $L$ with $\mathbf{R}^{m}$. Since $\nu$ satisfies (2.14) but not (2.16), then $(2.15)$ cannot hold. Namely, there must exist a ball $B^{\prime}$ centered in $\widetilde{\Sigma}$, and $r\left(B^{\prime}\right) \geq \delta^{\frac{1}{2}} r(B)$, such that $\beta_{\nu}^{m-1}\left(B^{\prime}\right) \leq \frac{1}{2} \epsilon$. We also have $B^{\prime} \subset \frac{1}{2} B$ by the same argument as in the proof of Theorem 2.16 in $[\mathrm{T}]$. Let $L^{\prime}$ be a best approximating $(m-1)$-plane for $\beta_{\nu}^{m-1}\left(B^{\prime}\right)$. We claim that the following holds:

$$
\Sigma \cap B^{\prime} \subset U_{\delta r(B)+\frac{\epsilon r\left(B^{\prime}\right)}{2}}\left(L^{\prime}\right),
$$

where $U_{\delta r(B)+\frac{\epsilon r\left(B^{\prime}\right)}{2}}\left(L^{\prime}\right)$ denotes the $\left(\delta r(B)+\frac{\epsilon r\left(B^{\prime}\right)}{2}\right)$-neighborhood of $L^{\prime}$. Indeed, we have

$$
\beta_{\nu}^{m-1}\left(B^{\prime}\right) \leq \frac{\epsilon}{2}
$$


Suppose $z \in \Sigma \cap B^{\prime}$. Then $z=\pi(z)+\pi_{L^{\perp}}(z)$, where $\pi$ and $\pi_{L^{\perp}}$ are the projections onto $L$ and $L^{\perp}$ respectively. Since $\Sigma \cap B^{\prime} \subset \Sigma \cap B$ and $\beta_{\mu}^{m}(B) \leq \delta$, we get $\left|\pi_{L^{\perp}}(z)\right| \leq \delta r(B)$. Moreover, by (3.10), $\operatorname{dist}\left(\pi(z), L^{\prime}\right) \leq \frac{\epsilon}{2} r\left(B^{\prime}\right)$. Thus, for all $z \in \Sigma \cap B^{\prime}$,

Therefore

$$
\operatorname{dist}\left(z, L^{\prime}\right) \leq|z-\pi(z)|+\operatorname{dist}\left(\pi(z), L^{\prime}\right) \leq \delta r(B)+\frac{\epsilon}{2} r\left(B^{\prime}\right)
$$

$$
\beta_{\mu}^{m-1}\left(B^{\prime}\right) \leq \frac{\delta r(B)+\frac{\epsilon}{2} r\left(B^{\prime}\right)}{r\left(B^{\prime}\right)} \leq \delta^{\frac{1}{2}}+\frac{\epsilon}{2} \leq \epsilon .
$$

We now prove (3.8). Pick $z_{0} \in \frac{1}{2} B \cap \widetilde{\Sigma}, r_{0}, r$ such that $\delta^{\frac{1}{2}} r(B) \leq r_{0}<r \leq r(B)$. Choose any $x \in \widetilde{\Sigma} \cap B\left(z_{0}, r_{0}\right)$, and let $z_{1}, x_{1} \in \Sigma \cap B$ be such that $\pi\left(z_{1}\right)=z_{0}, \pi\left(x_{1}\right)=$ $x$. Then

$$
\left|\frac{x-z_{0}}{r_{0}} R_{r_{0}, r} \nu\left(z_{0}\right)\right| \leq A+B+C
$$

where

$$
\begin{aligned}
& A=\left|\frac{x-z_{0}}{r_{0}} \cdot\left(R_{r_{0}, r} \nu\left(z_{0}\right)-R_{r_{0}, r} \mu\left(z_{1}\right)\right)\right|, \\
& B=\left|\frac{\left(x-z_{0}\right)-\left(x_{1}-z_{1}\right)}{r_{0}} \cdot R_{r_{0}, r} \mu\left(z_{1}\right)\right|
\end{aligned}
$$

and

$$
C=\left|\frac{x_{1}-z_{1}}{r_{0}} \cdot R_{r_{0}, r} \mu\left(z_{1}\right)\right| .
$$

Estimating $A$. Let us first estimate (3.12). We denote the kernel of the Riesz transform by $K$, and the annulus in $\mathbf{R}^{d}$ by $A\left(z_{0}, r_{0}, r\right)=\left\{y \in \mathbf{R}^{d} ; r_{0}<\left|y-z_{0}\right| \leq r\right\}$, the annulus in $L$ by $A_{L}\left(z_{0}, r_{0}, r\right)$. Then, we can write

$$
R_{r_{0}, r} \nu\left(z_{0}\right)=\int_{B \cap \pi^{-1}\left(A_{L}\left(z_{0}, r_{0}, r\right)\right)} K\left(z_{0}-\pi(y)\right) d \mu(y)
$$

and

$$
\begin{aligned}
& \left|R_{r_{0}, r} \nu\left(z_{0}\right)-R_{r_{0}, r} \mu\left(z_{1}\right)\right| \\
& =\left|\int_{B \cap \pi^{-1}\left(A_{L}\left(z_{0}, r_{0}, r\right)\right)} K\left(z_{0}-\pi(y)\right) d \mu(y)-\int_{A\left(z_{1}, r_{0}, r\right)} K\left(z_{1}-y\right) d \mu(y)\right| \\
& \leq S_{1}+S_{2} .
\end{aligned}
$$

where

$$
S_{1}:=\int_{B \cap \pi^{-1}\left(A_{L}\left(z_{0}, r_{0}, r\right)\right)}\left|K\left(z_{0}-\pi(y)\right)-K\left(z_{1}-y\right)\right| d \mu(y)
$$

and

$$
S_{2}:=\left|\int_{B \cap \pi^{-1}\left(A_{L}\left(z_{0}, r_{0}, r\right)\right)} K\left(z_{1}-y\right) d \mu(y)-\int_{A\left(z_{1}, r_{0}, r\right)} K\left(z_{1}-y\right) d \mu(y)\right| .
$$

Estimating $S_{1}$ from (3.15). To estimate $S_{1}$, we need the following intermediate estimates. First, note that if $y \in B \cap \pi^{-1}\left(A_{L}\left(z_{0}, r_{0}, r\right)\right) \cap \Sigma$, it follows from the fact that $\left|z_{0}-y\right|^{2}=\left|z_{0}-\pi(y)\right|^{2}+|\pi(y)-y|^{2}$ and $\pi(y) \in A_{L}\left(z_{0}, r_{0}, r\right)$ that the following holds

$$
\left|z_{0}-y\right| \geq\left|z_{0}-\pi(y)\right| \geq r_{0}
$$


Second, by (3.7),

$$
\left|\left(z_{0}-\pi(y)\right)-\left(z_{1}-y\right)\right| \leq\left|z_{0}-z_{1}\right|+|y-\pi(y)| \leq 2 \delta r(B) .
$$

Similarly, we claim that

(3.18) $\frac{1}{2}\left|z_{0}-y\right| \leq\left|z_{0}-\pi(y)\right| \leq 2\left|z_{0}-y\right| \quad$ and $\quad \frac{1}{2}\left|z_{0}-y\right| \leq\left|z_{1}-y\right| \leq 2\left|z_{0}-y\right|$.

Indeed, on one hand assuming $\delta$ small enough that $\delta r(B) \leq \frac{1}{2} r_{0}$,

$$
\begin{aligned}
\left|z_{0}-y\right| & \leq\left|z_{0}-\pi(y)\right|+|\pi(y)-y| \leq\left|z_{0}-\pi(y)\right|+\delta r(B) \\
& \leq\left|z_{0}-\pi(y)\right|+\frac{1}{2} r_{0} \leq 2\left|z_{0}-\pi(y)\right|, \quad \text { by }(3.16) .
\end{aligned}
$$

On the other hand

$$
\begin{aligned}
\left|z_{0}-\pi(y)\right| & \leq\left|z_{0}-y\right|+|y-\pi(y)| \\
& \leq\left|z_{0}-y\right|+\frac{1}{2} r_{0} \leq 2\left|z_{0}-y\right|, \quad \text { by (3.7). }
\end{aligned}
$$

The other estimate in (3.18) follows similarly. On one hand,

$$
\begin{aligned}
\left|z_{0}-y\right| & \leq\left|z_{0}-z_{1}\right|+\left|z_{1}-y\right| \leq \delta r(B)+\left|z_{1}-y\right| \\
& \leq\left|z_{0}-\pi(y)\right|+\left|z_{1}-y\right| \leq 2\left|z_{1}-y\right|, \quad \text { since } z_{0}=\pi\left(z_{1}\right) .
\end{aligned}
$$

On the other hand,

$$
\left|z_{1}-y\right| \leq\left|z_{1}-z_{0}\right|+\left|z_{0}-y\right| \leq \delta r(b)+\left|z_{0}-y\right| \leq 2\left|z_{0}-y\right| .
$$

Thus, to estimate $S_{1}$, noting that

$$
\begin{aligned}
\left|K\left(z_{0}-\pi(y)\right)-K\left(z_{1}-y\right)\right| \leq & \left|\frac{z_{0}-\pi(y)}{\left|z_{0}-\pi(y)\right|^{n+1}}-\frac{z_{1}-y}{\left|z_{0}-\pi(y)\right|^{n+1}}\right| \\
& +\left|\frac{z_{1}-y}{\left|z_{0}-\pi(y)\right|^{n+1}}-\frac{z_{1}-y}{\left|z_{1}-y\right|^{n+1}}\right| \\
= & : D_{1}+D_{2},
\end{aligned}
$$

one obtains on one hand, using (3.16) and (3.17),

$$
D_{1}=\frac{\left|z_{0}-\pi(y)-\left(z_{1}-y\right)\right|}{\left|z_{0}-\pi(y)\right|^{n+1}} \leq \frac{2^{n+1} C \delta r(B)}{\left|z_{0}-y\right|^{n+1}},
$$

and on the other hand, using (3.17) and (3.18),

$$
\begin{aligned}
D_{2} & =\left|z_{1}-y\right|\left|\frac{\left|z_{1}-y\right|^{n+1}-\left|z_{0}-\pi(y)\right|^{n+1}}{\left|z_{1}-y\right|^{n+1}\left|z_{0}-\pi(y)\right|^{n+1}}\right| \\
& \leq C_{n} \frac{\left|z_{0}-\pi(y)\right|^{n+1}}{\left|z_{0}-y\right|^{2 n+2}} \cdot|| z_{1}-y|-| z_{0}-\pi(y)|| . \\
& \leq C_{n} \frac{\delta r(B)}{\left|z_{0}-y\right|^{n+1}} .
\end{aligned}
$$


Putting (3.20) and (3.21) together gives

$$
\begin{aligned}
S_{1} & \leq \int_{B \cap \pi^{-1}\left(A_{L}\left(z_{0}, r_{0}, r\right)\right)} \frac{c \delta r(B)}{\left|z_{0}-y\right|^{n+1}} d \mu(y) \\
& \leq c \int_{\frac{1}{2} r_{0} \leq\left|y-z_{0}\right| \leq 2 r} \frac{\delta r(B)}{\left|z_{0}-y\right|^{n+1}} d \mu(y) \\
& \leq c \delta r(B) \int_{\frac{1}{2 r^{n+1}}}^{\left(\frac{2}{r_{0}}\right)^{n+1}} \mu\left(\left\{y, \frac{1}{\left|z_{0}-y\right|^{n+1}}>t\right\}\right) d t \\
& \leq c \delta r(B) \int_{\frac{1}{2 r^{n+1}}}^{\left(\frac{2}{r_{0}}\right)^{n+1}} \mu\left(B\left(z_{0}, t^{-\frac{1}{n+1}}\right)\right) d t \\
& \leq c \delta r(B) \int_{\left.\frac{1}{r_{0}}\right)^{n+1}} \frac{1}{t^{\frac{n}{n+1}}} d t \\
& \leq \frac{C^{\prime} \delta r(B)}{r_{0}} \leq 1 \quad \text { by choosing } \delta^{\frac{1}{2}}<C^{\prime-1} .
\end{aligned}
$$

Estimating $S_{2}$ from (3.15).

$$
\begin{aligned}
S_{2} & =\left|\int_{B \cap \pi^{-1}\left(A_{L}\left(z_{0}, r_{0}, r\right)\right)} K\left(z_{1}-y\right) d \mu(y)-\int_{A\left(z_{1}, r_{0}, r\right)} K\left(z_{1}-y\right) d \mu(y)\right| \\
& \leq \int_{B \cap\left(\pi^{-1}\left(A_{L}\left(z_{0}, r_{0}, r\right)\right) \triangle A\left(z_{1}, r_{0}, r\right)\right)}\left|K\left(z_{1}-y\right)\right| d \mu(y) .
\end{aligned}
$$

Now, we claim that for $\delta>0$ small enough, we have

$$
\Sigma \cap B \cap\left(\pi^{-1}\left(A_{L}\left(z_{0}, r_{0}, r\right)\right) \triangle A\left(z_{1}, r_{0}, r\right)\right) \subset A\left(z_{1}, \frac{1}{2} r_{0}, 2 r_{0}\right) \cup A\left(z_{1}, \frac{1}{2} r, 2 r\right) .
$$

We will only treat the case where $\pi(y) \in A\left(z_{0}, r_{0}, r\right)$ and $y \notin A\left(z_{1}, r_{0}, r\right)$. The other case follows in exactly the same manner. First, note that in the above case, either $\left|y-z_{1}\right| \leq r_{0}$, implying in particular that $\left|y-z_{1}\right| \leq 2 r_{0}$. Moreover, for such a $y$,

$$
\begin{aligned}
\left|y-z_{1}\right| & \geq\left|\pi(y)-z_{0}\right|-|y-\pi(y)|-\left|z_{1}-z_{0}\right| \geq r_{0}-2 \delta r(B) \quad \text { by }(3.7) \\
& \geq \frac{1}{2} r_{0},
\end{aligned}
$$

and hence, $y \in A\left(z_{1}, \frac{1}{2} r_{0}, 2 r_{0}\right)$. Otherwise, $\left|y-z_{1}\right|>r$ (a fortiori, $\left.\left|y-z_{1}\right|>\frac{1}{2} r\right)$ and

$$
\left|y-z_{1}\right| \leq|y-\pi(y)|+\left|\pi(y)-z_{0}\right|+\left|z_{1}-z_{0}\right| \leq 2 \delta r(B)+r \leq 2 r
$$

implying $y \in A\left(z_{1}, \frac{1}{2} r, 2 r\right)$. Hence,

$$
\Sigma \cap B \cap\left(\pi^{-1}\left(A_{L}\left(z_{0}, r_{0}, r\right)\right) \cap A\left(z_{1}, r_{0}, r\right)^{C}\right) \subset A\left(z_{1}, \frac{1}{2} r_{0}, 2 r_{0}\right) \cup A\left(z_{1}, \frac{1}{2} r, 2 r\right) .
$$

Using (3.23), we obtain

$$
\begin{aligned}
S_{2} & \leq \int_{A\left(z_{1}, \frac{1}{2} r_{0}, 2 r_{0}\right)} \frac{1}{\left|z_{1}-y\right|^{n}} d \mu(y)+\int_{A\left(z_{1}, \frac{1}{2} r, 2 r\right)} \frac{1}{\left|z_{1}-y\right|^{n}} d \mu(y) \\
& \leq 2^{n} \frac{\mu\left(B\left(z_{1}, 2 r_{0}\right)\right)}{r_{0}^{n}}+2^{n} \frac{\mu\left(B\left(z_{1}, 2 r\right)\right)}{r^{n}} \leq C_{n} .
\end{aligned}
$$


The estimates (3.22) and (3.24) combined give

$$
\left|\frac{x-z_{0}}{r_{0}} \cdot\left(R_{r_{0}, r} \nu\left(z_{0}\right)-R_{r_{0}, r} \mu\left(z_{1}\right)\right)\right| \leq\left|\frac{x-z_{0}}{r_{0}}\right| \cdot C \leq C^{\prime} .
$$

Estimating $B$. Let us now estimate (3.13): first note that

$$
\left|\left(x-z_{0}\right)-\left(x_{1}-z_{1}\right)\right| \leq\left|x-x_{1}\right|+\left|z_{0}-z_{1}\right| \leq 2 \delta r(B) \text { by }(3.7) .
$$

Moreover,

$$
\begin{aligned}
\left|R_{r_{0}, r} \mu\left(z_{1}\right)\right| & \leq \int_{r_{0}<\left|y-z_{1}\right| \leq r} \frac{1}{\left|y-z_{1}\right|^{n}} d \mu(y) \\
& \leq \int_{r^{-n}}^{r_{0}^{-n}} \mu\left(\left\{y:\left|K\left(y-z_{1}\right)\right|>t^{-n}\right\}\right) d t \\
& \leq \int_{r^{-n}}^{r_{0}^{-n}} \frac{1}{t} d t \leq C \log \left(\frac{r(B)}{r_{0}}\right) \leq C^{\prime}|\log (\delta)| .
\end{aligned}
$$

Therefore, assuming $\delta$ is small enough,

$$
\begin{aligned}
\left|\frac{\left(x-z_{0}\right)-\left(x_{1}-z_{1}\right)}{r_{0}} \cdot R_{r_{0}, r} \mu\left(z_{0}\right)\right| & \leq C \frac{\delta r(B)}{r_{0}}|\log \delta| \\
& \leq C \delta^{\frac{1}{2}}|\log \delta| \leq C
\end{aligned}
$$

Estimating $C$. Finally, we estimate (3.14): we want to apply Lemma 3.1 to evaluate $\left|\frac{x_{1}-z_{1}}{r_{0}} \cdot R_{r_{0}, r} \mu\left(z_{1}\right)\right|$. But we do not have $x_{1} \in B\left(z_{1}, r_{0}\right)$. Nevertheless, we have

$$
\left|x_{1}-z_{1}\right| \leq\left|x_{0}-z_{0}\right|+\left|z_{0}-z_{1}\right|+\left|x_{0}-x_{1}\right| \leq\left|x_{0}-z_{0}\right|+2 \delta r(B) \leq 2 r_{0} .
$$

Using (3.27), and applying Lemma 3.1 to the first term, we have

$$
\begin{aligned}
\left|\frac{x_{1}-z_{1}}{r_{0}} \cdot R_{r_{0}, r} \mu\left(z_{1}\right)\right| & \leq 2\left|\frac{x_{1}-z_{1}}{2 r_{0}} \cdot R_{2 r_{0}, r} \mu\left(z_{1}\right)\right|+\left|\frac{x_{1}-z_{1}}{r_{0}} \cdot R_{r_{0}, 2 r_{0}} \mu\left(z_{1}\right)\right| \\
& \leq 2 c+\left|\frac{x_{1}-z_{1}}{r_{0}}\right| \cdot\left|R_{r_{0}, 2 r_{0}} \mu\left(z_{1}\right)\right| .
\end{aligned}
$$

To estimate the second term on the right hand side of the inequality in (3.28), we simply notice that

$$
\left|R_{r_{0}, 2 r_{0}} \mu\left(z_{1}\right)\right| \leq r_{0}^{-n} \mu\left(B\left(z_{1}, 2 r_{0}\right)\right) \leq \tilde{c} .
$$

This implies the uniform boundedness of $C$.

Combining our estimates in (3.25), (3.26), (3.28), and (3.29), we have proven the claim we had set out to prove: namely, that for all $z_{0} \in \frac{1}{2} B \cap \widetilde{\Sigma}$, and $r_{0}, r$ with $\delta^{\frac{1}{2}} r(B) \leq r_{0} \leq r \leq r(B)$, if $\delta$ is small enough,

$$
\left|\frac{x-z_{0}}{r_{0}} \cdot R_{r_{0}, r} \nu\left(z_{0}\right)\right| \leq c, \quad \text { for } x \in \widetilde{\Sigma} \cap B\left(z_{0}, r_{0}\right) .
$$

Theorem 3.3. Let $\mu$ be a uniformly distributed measure with $n=\operatorname{dim}_{0} \mu, K$ a compact set. For every $\epsilon>0$, there exists some $\tau>0$ such that every ball $B$ centered in $\Sigma$ and contained in $K$, contains another ball $B^{\prime}$ also centered in $\Sigma$, which satisfies $\beta_{\mu}^{n}\left(B^{\prime}\right) \leq \epsilon$, and $r\left(B^{\prime}\right) \geq \tau r(B)$. Moreover, $\tau$ only depends on $\epsilon, K, n$ and $d$. 
Proof. We just apply Lemma $3.2(d-n)$ times. Indeed, since $\beta_{\mu}^{(d)}(B)=0$, there exists $B_{1} \subset B, \beta_{\mu}^{(d-1)}\left(B_{1}\right) \leq \epsilon_{1}, r\left(B_{1}\right) \sim r(B)$. By induction, we get a ball $B_{d-n} \subset B$, $r\left(B_{d-n}\right) \sim r(B), \beta_{\mu}^{(n)}\left(B_{d-n}\right) \leq \epsilon_{d-n}$. Making the successive $\epsilon_{i}$ 's as small as needed, since there are only finitely many steps, letting $\epsilon=\epsilon_{d-n}$, and $B^{\prime}=B_{d-n}$, we get $\epsilon>0, B^{\prime} \subset B, r\left(B^{\prime}\right) \sim r(B)$ such that $\beta_{\mu}^{(n)}\left(B^{\prime}\right) \leq \epsilon$.

\section{Stability of the $\beta$-numbers}

In the following section, we will write $\beta(B)$ for $\beta^{n}(B)$.

Lemma 4.1. Let $\mu$ be a Radon measure, $\Sigma$ its support. Let $\mu_{x, r}$ be the following measure (and $\Sigma_{x, r}$ its support):

$$
\mu_{x, r}(A)=\mu(r A+x)
$$

Then we have

$$
b \beta_{\mu_{x, r}}(B)=b \beta_{\mu}(r B+x)
$$

Proof. It is easily seen that $\Sigma=r \Sigma_{x, r}+x$. Let $L$ be an $n$-plane. Hence,

Let $y \in \Sigma_{x, r} \cap B$. Then $y^{\prime}=r y+x$ is in $\Sigma \cap B_{x, r}$ and $\operatorname{dist}(y, L)=\frac{1}{r} \operatorname{dist}\left(y^{\prime}, L+x\right)$.

$$
\sup _{\Sigma_{x, r} \cap B} \operatorname{dist}(y, L)=\frac{1}{r} \sup _{\Sigma \cap B_{x, r}} \operatorname{dist}\left(y^{\prime}, L+x\right) .
$$

On the other hand, let $p \in L \cap B$. Then $\operatorname{dist}\left(p, \Sigma_{x, r}\right)=\frac{1}{r} \operatorname{dist}(r p+x, \Sigma)$, where $r p+x=p^{\prime}, p^{\prime} \in(L+x) \cap B_{x, r}$. Hence,

$$
\sup _{L \cap B} \operatorname{dist}\left(p, \Sigma_{x, r}\right)=\sup _{L+x \cap B_{x, r}} \operatorname{dist}\left(p^{\prime}, \Sigma\right) .
$$

Adding (4.2) and (4.3), and taking the infimum over all $n$-planes proves (4.1).

We can now prove the following theorem. It states that the flatness of $\mu$ on a fixed number of bigger scales than $B$ implies flatness at scale $B$. Its proof is analogous to the proof of Lemma [3.6] in [T].

Lemma 4.2. Let $\mu$ be a Radon measure on $\mathbf{R}^{d}$ that is uniformly distributed with $\operatorname{dim}_{0}(\mu)=n$ and $K$ a compact set in $\mathbf{R}^{d}$. Let $\epsilon>0$, and $\delta_{0}$ be $\tau_{0}$ from Theorem 2.11 . There exists an integer $N>0$, depending only on $n, \epsilon, d$ and $K$ such that for every ball $B$ centered in $\Sigma \cap K$, if

$$
2^{N} B \subset K, \quad \beta_{\mu}\left(2^{k} B\right) \leq \frac{\delta_{0}}{4}, \quad 1 \leq k \leq N
$$

then

$$
b \beta_{\mu}(B) \leq \epsilon
$$

Proof. We argue by contradiction. Suppose there is no such $N$. Then, for every $j$, there exist a $n$-AOD measure $\mu_{j}$, a ball $B_{j}=B\left(x_{j}, r_{j}\right), x_{j} \in K \cap \Sigma, 2^{j} r_{j} \leq \operatorname{diam}(K)$ such that

but

$$
\beta_{\mu_{j}}\left(2^{k} B_{j}\right) \leq \frac{\delta_{0}}{4}, \quad 1 \leq k \leq j
$$

$$
b \beta_{\mu_{j}}\left(B_{j}\right) \geq \epsilon
$$


Note that $x_{j} \in \Sigma \cap K, 2^{j} r_{j} \leq \operatorname{diam}(K)$ imply that, passing to a subsequence, $r_{j} \rightarrow 0$ and $x_{j} \rightarrow x, x \in K$, as $j \rightarrow \infty$. Now, let $\nu_{j}$ be the measure defined as

$$
\nu_{j}(A)=\frac{\mu_{j}\left(r_{j} A+x_{j}\right)}{\mu_{j}\left(B_{j}\right)} .
$$

There exists some subsequence of $\mu_{j}$ that converges weakly to a measure $\nu$ as $j \rightarrow \infty$. Indeed, for any ball $B(0, R), R>1$, if $\phi_{j}$ is the distribution function of $\mu_{j}$ we have

$$
\frac{\mu_{j}\left(r_{j} B(0, R)+x_{j}\right)}{\mu_{j}\left(B_{j}\right)}=\frac{\phi_{j}\left(R r_{j}\right)}{\phi_{j}\left(r_{j}\right)} \leq 5^{d} R^{d},
$$

according to Theorem 2.4. Since each $\nu_{j}$ is uniformly distributed, $\nu$ is also uniformly distributed. Moreover, since the $\mu_{j}$ 's are doubling with the same constant, using Lemma 2.9 and (4.4),

$$
2 b \beta_{\nu}(B(0,2)) \geq \limsup _{j \rightarrow \infty} b \beta \nu_{j}(B(0,1))=\limsup _{j \rightarrow \infty} b \beta_{\mu_{j}}\left(B_{j}\right) \geq \epsilon .
$$

On the other hand, for all $k \geq 0$, by (4.4),

$$
\beta_{\nu}\left(B\left(0,2^{k-1}\right)\right) \leq 2 \liminf _{j \rightarrow \infty} \beta_{\nu_{j}}\left(B\left(0,2^{k}\right)\right)=2 \liminf _{j \rightarrow \infty} \beta_{\mu_{j}}\left(2^{k} B_{j}\right) \leq \frac{\delta_{0}}{2} .
$$

Let $\lambda$ be the tangent measure of $\nu$ at $\infty$. Define $\nu_{k}$ in the following manner:

$$
\nu_{k}(A)=\frac{\nu\left(2^{k} A\right)}{2^{n k}}
$$

Then $\nu_{k} \rightarrow \lambda$ and

$$
\beta_{\lambda}(B(0,1)) \leq 2 \liminf _{k \rightarrow \infty} \beta_{\nu_{k}}(B(0,2))=2 \liminf _{k \rightarrow \infty} \beta_{\nu}\left(B\left(0,2^{k+1}\right) \leq \delta_{0} .\right.
$$

By Theorem 2.11, this implies that $\nu$ is flat, contradicting $b \beta_{\nu}(B(0,1))>\epsilon$.

We prove that a uniformly distributed measure is doubling and asymptotically optimally doubling.

Lemma 4.3. Suppose $\mu$ is a uniformly distributed Radon measure in $\mathbf{R}^{d}$ with $\operatorname{dim}_{0} \mu=n, \operatorname{dim}_{\infty} \mu=p$ and such that $\mu(B(x, r))=\phi(r)$, for $x \in \Sigma$. Then $\mu$ is doubling and asymptotically optimally $n$-doubling.

Proof. We first prove that $\mu$ is doubling. This follows easily from Theorem 2.4. Indeed, if $x \in \operatorname{supp}(\mu), r>0$,

$$
\mu(B(x, 2 r)) \leq 10^{d} \mu(B(x, r)) .
$$

To prove that $\mu$ is $n$-asymptotically optimally doubling, let $K$ be a compact set such that $K \cap \Sigma \neq \emptyset$, and $\tau \in(0,1)$. Choose $x$ in $K \cap \Sigma$. Then

$$
\frac{\mu(B(x, \tau r))}{\mu(B(x, r))}=\frac{\phi(\tau r)}{\phi(r)}
$$

and hence, using Theorem 2.2,

$$
\begin{aligned}
\lim _{r \rightarrow 0} \sup _{x \in K \cap \Sigma} \frac{\mu(B(x, \tau r))}{\mu(B(x, r))} & =\lim _{r \rightarrow 0} \inf _{x \in K \cap \Sigma} \frac{\mu(B(x, \tau r))}{\mu(B(x, r))}=\lim _{r \rightarrow 0} \frac{\phi(\tau r)}{\phi(r)} \\
& =\lim _{r \rightarrow 0} \frac{\phi(\tau r)}{\tau^{n} r^{n}} \frac{r^{n}}{\phi(r)} \tau^{n}=\tau^{n} .
\end{aligned}
$$

The proof of the following corollary is analogous to the proof of Theorem [4.2] in [PTT] since uniformly distributed measures are $n$-AOD and doubling. 
Corollary 4.4. Let $\mu$ be a uniformly distributed measure on $\mathbf{R}^{d}, K$ compact set in $\mathbf{R}^{d}$. Fix $\eta>0$. Then there exists $\delta>0$ depending on $\eta, \mu, n$ and $d$ such that if $B$ is a ball centered in $\Sigma \cap K$, with $\beta_{\mu}(B)<\delta$, then $b \beta_{\mu}\left(B^{\prime}\right) \leq \eta$ for any ball $B^{\prime} \subset \frac{1}{2} B$ centered in $\Sigma$.

We can finally prove Theorem 1.3. The proof is identical to the proof of Theorem $[1.2]$ in $[\mathrm{T}]$. We repeat it for the reader's convenience.

Proof. Let $K$ be compact, $\epsilon>0$. By Theorems 3.3 and 4.4, there exists $c$ depending on $\epsilon$ and $K$ such that any ball centered in $\Sigma \cap K$ contains a ball $B^{\prime}$, $r\left(B^{\prime}\right) \geq \operatorname{cr}(B)$ contained in $B$ such that every ball $B^{\prime \prime}$ centered in $\Sigma \cap K$ and contained in $\frac{1}{2} B^{\prime}$ satisfies $b \beta_{\mu}\left(B^{\prime \prime}\right)<\epsilon$. An application of Theorem [15.2] from [DT] gives the result.

\section{References}

[DKT] David, G., C. E. Kenig, and T. Toro: Asymptotically optimally doubling measures and Reifenberg flat sets with vanishing constant. - Comm. Pure Appl. Math. 54, 2001, 385-449

[Del] De Lellis, C.: Rectifiable sets, densities, and tangent measures. - Zur. Lect. Adv. Math., Eur. Math. Soc., Zurich, 2008

[DS1] David, G., and S. Semmes: Singular integrals and rectifiable sets in $\mathbf{R}^{n}$. - Asterisque 193, 1991.

[DS2] David, G., and S. Semmes: Analysis of and on uniformly rectifiable sets. - Math. Surveys Monogr. 38, Amer. Math. Soc., Providence, RI, 1993.

[DT] David, G., and T. Toro: Reifenberg parametrizations for sets with holes. - Mem. Amer. Math. Soc. 215, Providence, RI, 2012.

[KiP] Kirchheim, B., and D. Preiss: Uniformly distributed measures in Euclidean spaces. Math. Scand. 90, 2002, 152-160.

[KoP] Kowalski, O., and D. Preiss: Besicovitch-type properties of measures and submanifolds. - J. Reine Angew. Math. 379, 1987, 115-151.

[M] Mattila, P.: Geometry of sets and measures in Euclidean spaces. - Cambridge Stud. Adv. Math. 44, Cambridge Univ. Press, Cambridge, 1995.

[P] Preiss, D.: Measures in $\mathbf{R}^{n}$ : distribution, rectifiability, and densities. - Ann. of Math. (2) $125,1987,537-643$.

[PTT] Preiss, D., X. TOlsA, and T. Toro: On the smoothness of Hölder doubling measures. Calc. Var. Partial Differential Equations 35:3, 2009, 339-363.

[T] Tolsa, X.: Uniform measures and uniform rectifiability. - J. Lond. Math. Soc. (2) 92, 2015, $1-18$.

Received 1 May 2018 • Accepted 6 September 2018 